\title{
Surgical Closure of Ischemic Ventricular Septal Rupture: a case report
}

\author{
Hassan Sandogij ${ }^{1}$, Mohammed Talat ${ }^{2}$ and Yasser Mubarak ${ }^{3 *}$ \\ ${ }^{1}$ Head Department of Cardiac Surgery, Madinah Cardiac Center, King Fahad Hospital, Madinah, KSA \\ ${ }^{2}$ National Heart Institutions, Cairo, Egypt; Madinah Cardiac Center, King Fahad Hospital, Madinah, KSA \\ ${ }^{3}$ Cardiothoracic Surgery Department, Faculty of Medicine, Minia University, Egypt; Madinah Cardiac Center, King Fahad Hospital, Madinah, KSA
}

\begin{abstract}
Abbreviations
VSR/D: ventricular septal rupture/ defect; AMI: acute myocardial infarction; STEMI: ST segment elevation MI; CAD: coronary artery disease; CABG: coronary artery bypass grafting; PCI: percutaneous coronary intervention; IABP: intra-aortic balloon pump; LAD: left anterior descending artery; CX: circumflex; RCA: right coronary artery; PDA: posterior descending artery; MVD: multivessel disease; TTE: trans thoracic echo; CPB: cardiopulmonary bypass; EF: ejection fraction; CHF: congestive heart failure; LCO: low cardiac output; MOF: multi organ failure; MR: mitral regurgitation; TR: tricuspid regurgitation; LV: left ventricle; RV: right ventricle; LVEDP: left ventricular end diastolic pressure; POD: post-operative day.
\end{abstract}

\section{Introduction}

VSR (Post-MI) incidence is 1-2\%. Early post-infarction interventions like; thrombolytic therapy, primary PCI, and urgent CABG decrease its incidence. Anterior MI causes apical or anteroseptal VSR (2/3), and posterior MI causes basal or posteroseptal VSR (1/3) [1-5].

VSR developed from hours - 2 weeks after AMI. It is caused by complete obstruction of a single coronary artery with poor collaterals. The prognosis of untreated VSR is extremely poor. Congestive heart failure (CHF), cardiogenic shock developed early leading to death. Posterior MI may be associated with severe mitral regurgitation (MR) leading to CHF, and pulmonary edema. The degree of left-right shunt depends on its size and pressure gradient across the septum. If VSR is large, right ventricle (RV) cannot tolerate sudden increase in volume load, and develops RV failure. So, severe biventricular failure is not uncommon with VSR [5].

\section{Case Report}

A 72-years old male complaining of sudden onset chest pain was referred to our cardiac centre. ECG was revealed acute inferior STsegment elevation (STEMI). Relatives denied previous history of any chest pain or CCU admission. The patient was a ex-smoker, diabetic (type I), hypertension with a family history of coronary heart disease (CAD). On physical examination, the patient was restless, and distress. The blood pressure was $80 / 50 \mathrm{~mm} \mathrm{Hg}$, pulse $125 \mathrm{bpm}$, respiratory rate $24 \mathrm{bpm}$. His skin was cool and poorly perfused with shallow respiration, and weak peripheral pulses. Laboratory investigations were showing troponin $(0.170 \mathrm{ng} / \mathrm{mL})$, serum creatinine level $(1.3 \mathrm{mg} / \mathrm{dL})$, and MBCK level (229 U/L). Coronary angiography was revealed total occluded left descending artery (LAD) and right coronary artery (RCA), and primary PCI was performed. Trans-Thoracic Echography (TTE) demonstrated a VSR $(20 \mathrm{~mm})$ located inferior basal and $\mathrm{EF}<25 \%$ (Figures 1 and 2). Patient was in cardiogenic shock, and intra-aortic balloon pump (IABP) was inserted. Chest X-ray showed increased cardiothoracic ratio with lung congestion. Medical management is aimed to improve cardiac output and reduce shunt. Inotropes, diuretics, and IABP are often used. Patient is hemodynamically stable and still on IABP for 14-days before surgery. Heparin is stopped 6-hours before surgery.

Patient was urgently transferred to operative room. Median sternotomy, then cardiopulmonary bypass (CPB) with moderate hypothermia $\left(32^{\circ} \mathrm{C}\right)$ was established after cannulation of aorta, SVC, and IVC with tapes around cavae. IABP was stopped then aortic crossclamp was placed, and antegrade blood cardioplegic arrest was induced. We made longitudinal incision lateral and parallel to left descending artery (LDA) in left ventricle infarct area. VSR identified in basal apical septum. Stay sutures were placed to expose the edges of the defect (Figure 3). The necrotic tissue was debrided, revealing a defect 2.5 $\mathrm{cm}$. VSR closure without tension employed using a bovine pericardial patch with Teflon felt pledgets placed on RV (Figure 4). Ventriculotomy was closed by double layer buttressed with Teflon felt strips (Figure 5). Tisseel Lyo Sealant used after preparation by Fibrinotherm apparatus to ensure complete hemostasis (Figures 6 and 7). TTE revealed no residual shunt. Patient weaned from CPB smoothly with minimal inotropes and IABP. IABP weaned after 2 days. Patient was discharged from hospital on 10th POD without any event.

\section{Discussion}

VSR is characterized by poor ventricular function. Ischemia caused by aortic cross clamp, and ischemic reperfusion injury has a poor outcome. Adequate myocardial protection during surgery is considered to be the cornerstone for a better outcome [6]. So that surgery is very high risk, and tries to do the best during myocardial preservation.

${ }^{\star}$ Correspondence to: Mubarak Y, MD. Assistant Professor of Cardiothoracic surgery, Minia University, Egypt. Associate Consultant of Cardiac Surgery, Madinah Cardiac Center, KSA, Tel: +201002554078/+966560708223; E-mail: yassermubarak73@gmail.com

Key words: Ventricular Septal Rupture, Ischemic Ventricular Septal Defect, Acute Myocardial Infarction, Post Infarction Mechanical Complications

Received: April 25, 2021; Accepted: May 25, 2021; Published: May 28, 2021 


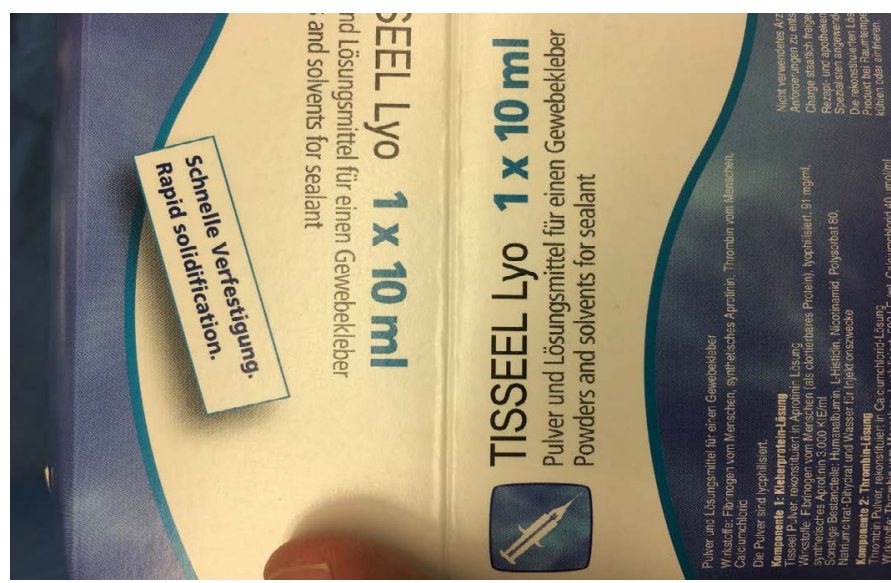

Figure 1. Pre-operative echocardiogram shows a ventricular septal rupture

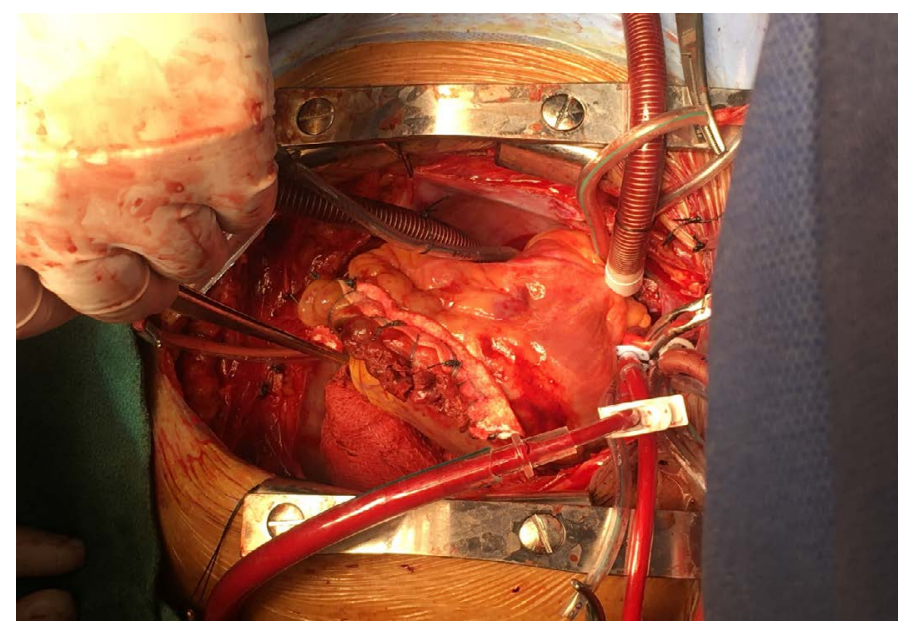

Figure 2. Pre-operative echocardiogram shows a ventricular septal rupture with left-to righ shunt flow

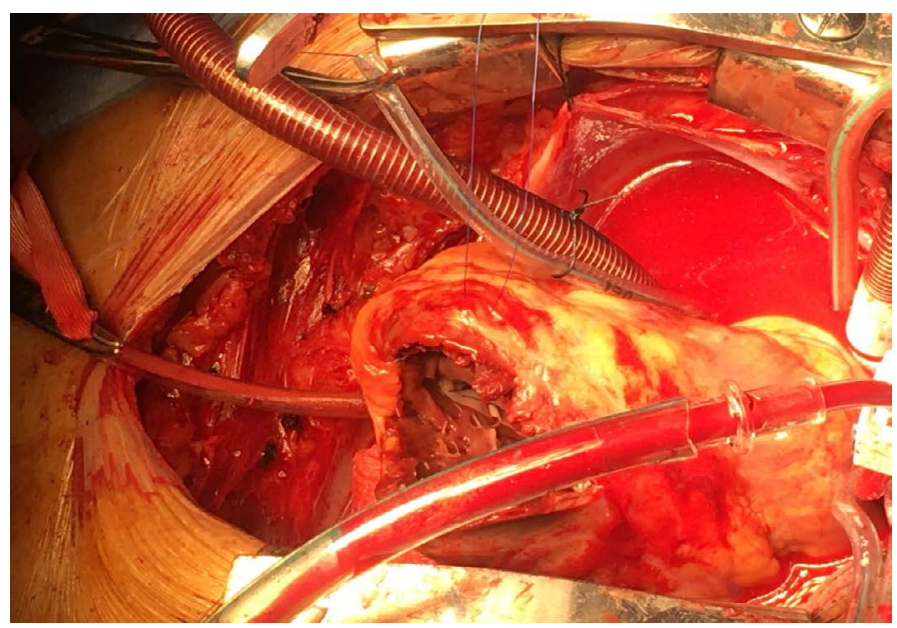

Figure 3. Intraoperative Photo showed VSR through Left Ventricular incision

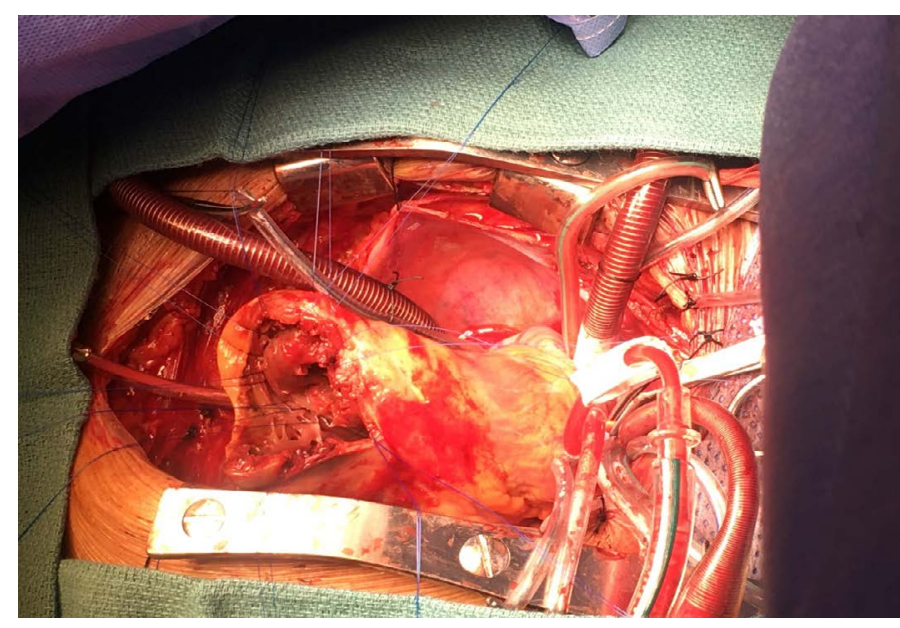

Figure 4. Intraoperative VSR closed by bovine pericardial patch

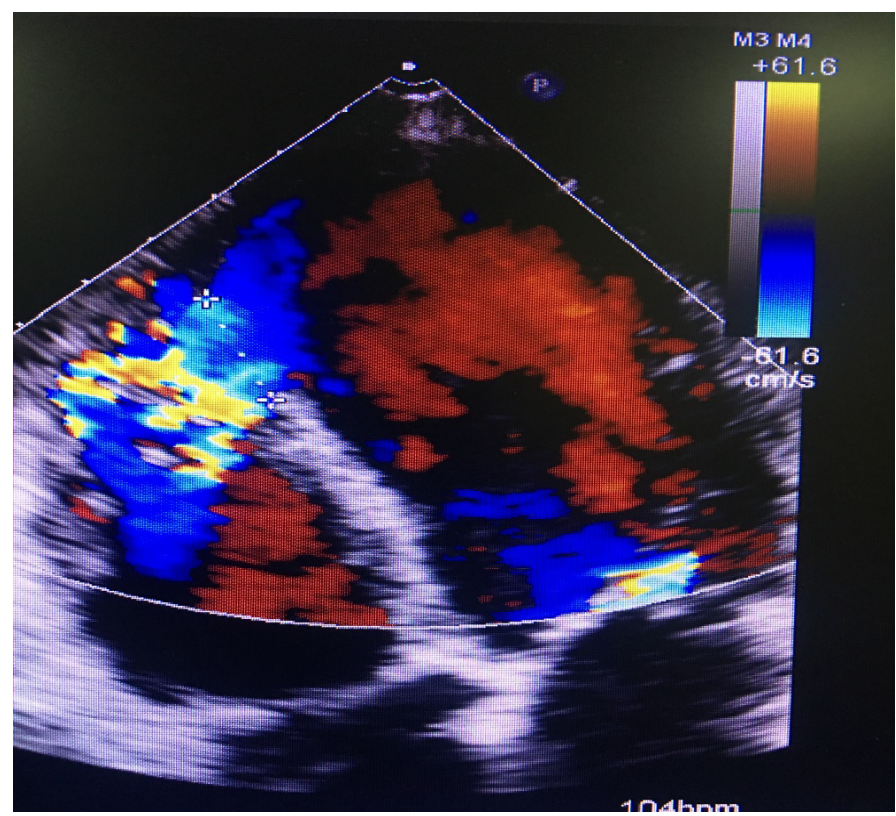

Figure 5. Linear closure oh left ventricular incision by two Teflon strips

VSR is a mechanical complication of MI with $50 \%$ surgical mortality. Many factors contribute to poor surgical outcome like; emergency, MVD, posterior VSR, incomplete revascularization, intractable shock and MOF [6]. In our case, patient was hemodynamically stable and still on IABP for two weeks.

VSR associated with cardiogenic shock has a very bad prognosis. Achieving hemodynamic stability before surgery may be beneficial, however prolonged attempts to improve patients' hemodynamics have drawback on delaying surgical intervention [2]. Preoperatively, high inotropics support and placement of IABP was essential to decrease left - right shunt, and MOF, and then surgical VSR closure done. 


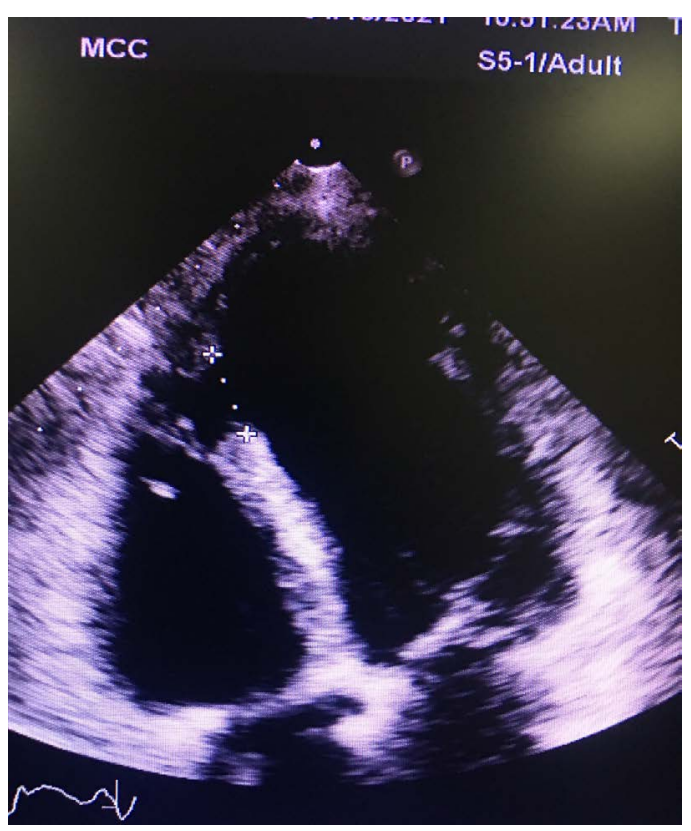

Figure 6. Hemostasis with Tisseel Lyo sealant

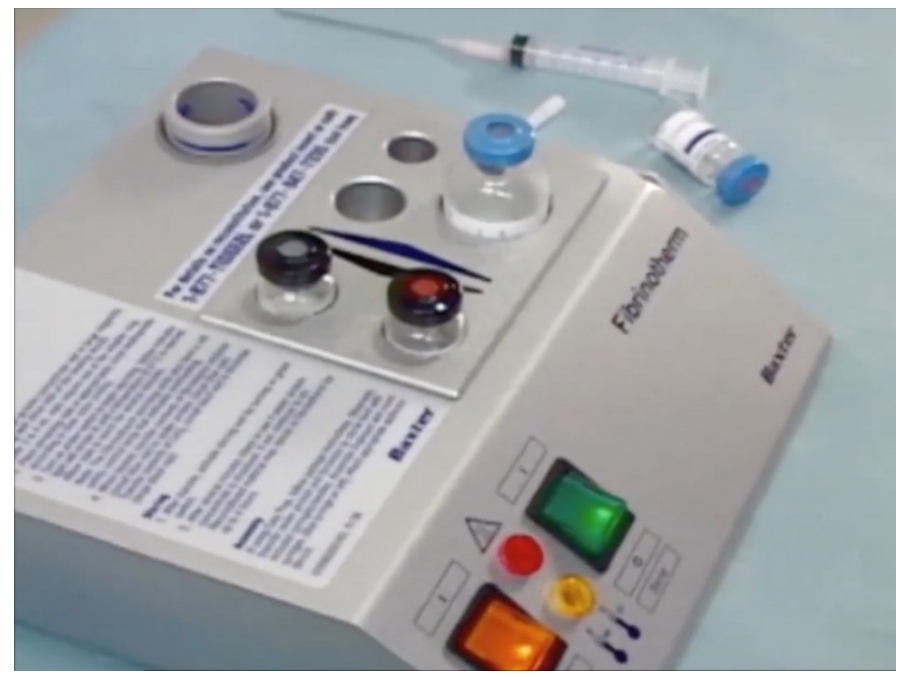

Figure 7. Fibrinotherm appratus used to preparation of Tisseel sealant

VSR repair is still a challenging with a risk of residual shunt and high mortality. CABG can be done safely to control the risk of CAD; especially in MVD, it should be routinely done $[3,4]$. The role of primary PCI or CABG in cases of VSR closure is debatable, as myocardial damage is trans-mural with residual viability questionable. However, in our case PCI to LAD, and RCA was done.

\section{Conclusion}

Inspite post-infarction VSR has high surgical mortality, surgical closure is mandatory after optimizing medical and mechanical support.

\section{Declarations}

\section{Ethics approval and consent to participate}

Patient confirms that have read and understood the information about the research as provided in the participant information sheet inside his file. Ethical committee approved that case report for publication. Patient signed agreement to participate the research, accepted to publish imaging from operation. The study conformed to the principles of "Declaration of Helsinki" and the investigator followed the appropriate safeguards regarding the rights and welfare of the human participants that have been included in the performed study. The formal approval and permission from our Cardiac Center, and Heart Team Meeting was taken.

\section{Consent for publication}

It was obtained written consent from patients.

\section{Availability of data and material}

It is available from recording files and data at cardiothoracic surgery departments and cardiology clinics for follow up.

\section{Competing interest}

None to declare.

\section{Funding}

None.

\section{References}

1. Furukawa K, Shirasaki Y, Ishii H, Nakamura E, Nakamura K (2020) Extended sandwich technique via the right atrial approach for post-infarction posterior ventricular septal rupture. General Thorac Cardiovasc Surge 68: 629-632. [Crossref]

2. Deja MA, Szostek J, Widenka K, Szafron B, Spyt TJ, et al. (2000) Post infarction ventricular septal defect can we do better? Euro J Cardiothorac Surg 18: 194-201. [Crossref]

3. Labroussea L, Choukrouna E, Chevalierb JM, Madonnaa F, Robertiea F, et al. (2002) Surgery for post infarction ventricular septal defect (VSD): risk factors for hospital death and long-term results. Euro J Cardiothorac Surg 21: 725-732. [Crossref]

4. Barkera TA, Ramnarineb IR, Wood EB, Grayson AD, Aud J, et al. Repair of postinfarct ventricular septal defect with or without coronary artery bypass grafting in the northwest of England: a 5-year multi-institutional experience. Euro J Cardiothorac Surg 24: 940-946. [Crossref]

5. Gilmanov D, Farneti P, Solinas M, Glauber M (2013) Severe mitral regurgitation in patients with post-infarction inter-ventricular septal defect: a simple way of simultaneous valve repair. Euro J Cardio-Thorac Surg 43: 184-186. [Crossref]

6. Apostolakis E, Kallikourdis A, Baikoussis NG, Dedeilias P, Dougeni D. The nonischemic repair as a safe alternative method for repair of anterior post-infarction. Cardiothorac Surg 5: 6.
Copyright: (C2021 Sandogji H. This is an open-access article distributed under the terms of the Creative Commons Attribution License, which permits unrestricted use, distribution, and reproduction in any medium, provided the original author and source are credited. 